\title{
On the Interpolation of Reduced-Order Models
}

\section{Yao Yue, Lihong Feng, Peter Benner}

\section{Motivation: Existing PMOR Methods and Their Limitations}

Parametric Model Order Reduction (PMOR) is a popular technique in accelerating the parametric studies of large-scale systems. Parametric Reduced-Order Models (pROMs) can be built via:

(1) Projection onto Global Bases. For the large-scale parametric full-order model (FOM)

$$
(s \mathcal{I}-\mathcal{A}(p)) X=\mathcal{B}(p), \quad \mathcal{A}(p) \in \mathbb{R}^{n \times n}, \mathcal{B}(p) \in \mathbb{R}^{n \times 1},
$$$$
y=\mathcal{C}(p) X \text {, }
$$$$
\mathcal{C}(p) \in \mathbb{R}^{1 \times n}
$$

G $\mathbb{R}^{n \times k}$ that contain global information and build the $\mathrm{pROM}$

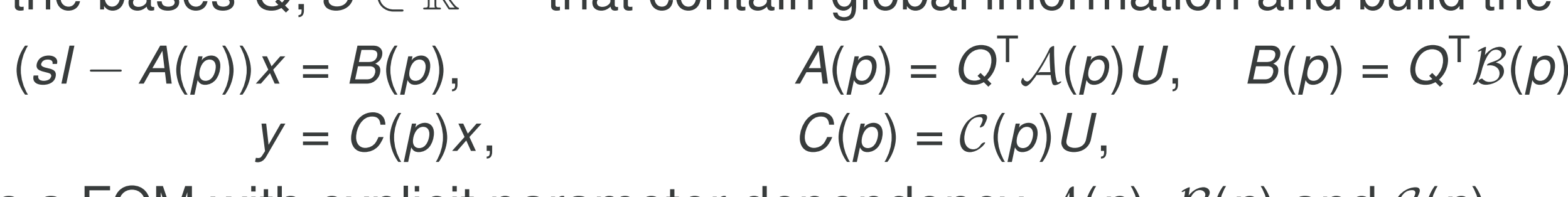

Requires a FOM with explicit parameter dependency $\mathcal{A}(p), \mathcal{B}(p)$ and $\mathcal{C}(p)$

To be efficient, the FOM must be in an affine form to pre-computate the expensive parts.

- Suffers from the curse of dimensionality.

2 Interpolate the local bases to obtain a parametric bases. For example, on the Stiefel manifold interpolate $Q\left(p_{1}\right), Q\left(p_{2}\right), \ldots, Q\left(p_{n_{p}}\right)$ to obtain $Q(p)$, and $U\left(p_{1}\right), U\left(p_{2}\right), \ldots, U\left(p_{n_{p}}\right)$ to obtain $U(p)$.

Requires a FOM with explicit parameter dependency.

- All bases have to be stored. All bases must be of the same dimension.

The heuristic of interpolation on the geodesic sometimes gives wrong results.

(3) Directly interpolate the nonparametric Reduced-Order Models (ROMs). An existing method:

make the ROMs consistent, and then interpolate them on the manifold of general linear group.

- Fails to give accurate results for our numerical example.

(4) Data-Driven Methods. Construct pROMs with samples in the frequency or time domain

e.g., the Loewner Framework. Suffers from the curse of dimensionality.

\section{The General Setting of the Pole-Matching PMOR Method}

We assume all data we know is $n_{p}$ ROMs:

$$
\left(s^{(i)}-A^{(i)}\right) X=B^{(i)}
$$$$
y=C^{(i)} x
$$

The $i$-th ROM built at $p$

We require: (1) all ROMs are accurate enough

2 we have sufficiently many ROMs to represent the parameter space.

We don't require the ROMs to be: $\mathbb{\otimes}$ built by the same methods; $\otimes$ of the same dimension.

\section{The Modified Modal Realization (MMR)}

For now, consider a single ROM, drop the index (i) in (1), and assume all eigenvalues to be simple.

For a real eigenpair $\left(\lambda_{j}, v_{j}\right)$ satisfying $A v_{j}=\lambda_{j} v_{j}$

we define $\quad \Lambda_{j}=\left[\lambda_{j}\right], \quad P_{j}=\left[v_{j}\right] \quad$ and $\quad m_{j}=1$

- For the conjugate complex eigenpairs $\left(a_{j} \pm ı b_{j}, r_{j} \pm ı q_{j}\right)$ satisfying $A\left(r_{j} \pm ı q_{j}\right)=\left(a_{j} \pm ı b_{j}\right)\left(r_{j} \pm ı q_{j}\right)$, we define $\quad \Lambda_{j}=\left[\begin{array}{cc}a_{j} & b_{j} \\ -b_{j} & a_{j}\end{array}\right], \quad P_{j}=\left[\begin{array}{ll}r_{j} & q_{j}\end{array}\right] \quad$ and $\quad m_{j}=2$

Define $\Lambda=\operatorname{diag}\left\{\Lambda_{1}, \Lambda_{2}, \ldots, \Lambda_{m}\right\}, P=\left[P_{1}, P_{2}, \ldots, P_{m}\right]$, and it follows $A=P \wedge P^{-1}$. Therefore

$$
y=C(s I-A)^{-1} B=C\left(s l-P \wedge P^{-1}\right)^{-1} B=C P(s I-\Lambda)^{-1} P^{-1} B=\sum_{j=1}^{m} C_{j}^{\prime}\left(s I-\Lambda_{j}\right)^{-1} B_{j}^{\prime}
$$

where $C^{1}=C P=\left[C_{1}^{1}, C_{2}^{1}, \ldots, C_{m}^{1}\right], B^{1}=P^{-1} B=\left[B_{1}^{1, T}, B_{2}^{1, T}, \ldots, B_{m}^{1, T}\right]^{\top}, C_{j}^{1} \in \mathbb{R}^{1 \times m_{j}}$, and $B_{j}^{\mid} \in \mathbb{R}^{m_{j} \times 1}$

\section{For the real eigenpair $\left(\lambda_{j}, v_{j}\right), C_{j}$ and $B_{j}$ are scalars.}

We define $C_{j}^{\prime \prime}=C_{j}^{\prime} B_{j}^{\mid} \quad$ and $\quad B_{j}^{\prime \prime}=1$ and derive $\quad C_{j}^{\prime}\left(s I-\Lambda_{j}\right)^{-1} B_{j}^{\prime}=\frac{C_{j}^{\prime} B_{j}^{1}}{s-\lambda_{j}}=C_{j}^{\prime \prime}\left(s l-\Lambda_{j}\right)^{-1} B_{j}^{\prime \prime}$

- For the conjugate complex eigenpairs $\left(a_{j} \pm ı b_{j}, r_{j} \pm I q_{j}\right)$, we first denote $C_{j}^{l}=\left[C_{j, 1}^{l}, C_{j, 2}^{!}\right] \in \mathbb{R}^{1 \times 2}$

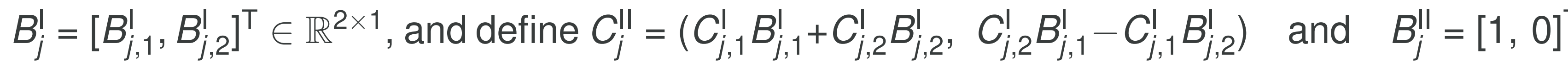

Then, we derive $C_{j}^{!}\left(s l-\Lambda_{j}\right)^{-1} B_{j}^{!}=\frac{C_{j}^{!}\left[\begin{array}{cc}s-a_{j} & b_{j} \\ -b_{j} & s-a_{j}\end{array}\right] B_{j}^{!}}{\left(s-a_{j}\right)^{2}+b_{j}^{2}}=C_{j}^{\prime \prime}\left(s I-\Lambda_{j}\right)^{-1} B_{j}^{\prime \prime}$,

Therefore, $\quad y=\sum_{j=1}^{m} C_{j}^{\prime}\left(s I-\Lambda_{j}\right)^{-1} B_{j}^{\prime}=\sum_{j=1}^{m} C_{j}^{\prime \prime}\left(s I-\Lambda_{j}\right)^{-1} B_{j}^{\prime \prime}=C^{\prime \prime}(s I-\Lambda)^{-1} B^{\prime \prime}$

where $C^{\|}=\left[C_{1}^{\|}, C_{2}^{\|}, \ldots, C_{m}^{\|}\right]$and $B^{\| \prime}=\left[B_{1}^{\|, T}, B_{2}^{\|, T}, \ldots, B_{m}^{\|, T}\right]^{\top}$

The Modified Modal Realization (MMR) for the system $(A, B, C)$ is defined as $\left(\Lambda, B^{\prime \prime}, C^{\prime \prime}\right)$.

- Physically Meaningful: equivalent to Pole-Residue Expansion. The nonzero entries of $\Lambda$ store the positions of the poles, $C^{\prime \prime}$ stores the amplitudes of the poles, no need to store $B^{\| \prime}$.

- Ideal for Interpolation. Interpolating the positions and amplitudes of the poles makes physical sense. Converting a ROM to MMR eliminates the freedom introduced by realization. - Efficient for Storage. For an order- $k$ system: we only need to store $2 k$ real numbers.

- Flexible. It is easy to: (1) change the order of the poles; 2 remove unimportant poles.

The Importance of Removing the Freedom Introduced by Realization

- $\left(\wedge^{(i)}, B^{1,(i)}, C^{1,(i)}\right)(i=1,2)$ are two equivalent ROMs of order 3 built for the same $p$ value. - $B^{1,(1)}=[16,2,1]^{\top}, B^{1,(2)}=[4,4,4]^{\top}, \Lambda^{(1)}=\Lambda^{(2)}=\operatorname{diag}\{1,2,3\}$,

$C^{\mathrm{l},(1)}=[1,8,16], \quad C^{\mathrm{l},(2)}=[4,4,4] . \quad$ All three poles have a magnitude of 16 .

A linear interpolation with equal weight 0.5 gives $B^{1,(3)}=[10,3,2.5]^{\top}, C^{\mathrm{l},(3)}=[2.5,6,10]$, with the amplitudes of the three poles to be 25,18 and 25 , respectively, which are all wrong.

\section{The Pole-Matching PMOR Method}

(1) Given $n_{p}$ ROMs built at $p_{1}, p_{2}, \ldots, p_{n_{p}}$ : convert all these ROMs into MMR.

2 To get the ROM for $p_{*}$, we first choose an interpolation algorithm and accordingly, the relevant given ROMs required for interpolation. Actually, they also define a region $\Omega^{\prime}$ within which we have a pROM of a uniform formulation.

(3) Match the positions and amplitudes of the poles

(4) Interpolate the positions and amplitudes of all matched poles to obtain the pROM valid on $\Omega^{\prime}$.

\section{Practical Considerations}

Pole-Matching Criterion. A merit function: weighted sum of the position-differences and the amplitude-differences of the poles.

The Case of Complex A. Simply conduct the complex eigenvalue decomposition to diagonalize $A$ and treat all eigenvalues as what we do for real eigenvalues in the case of real $A$.

Semisimple Eigenvalues. (1) The same multiplicity for all p's: treat it as a simple eigenvalue. 2 Otherwise: must "split" the multiplicity in pole-matching

(Nearly) Defective Eigenvalues. The current method is not applicable.

\section{A Reference: the Loewner Realization}

- The Loewner Framework builds ROMs from samples of the Frequency Transfer Function (FRF) Interpolating ROMs in the Loewner realization is equivalent to interpolating the FRF.

\section{Numerical Results and Conclusions}

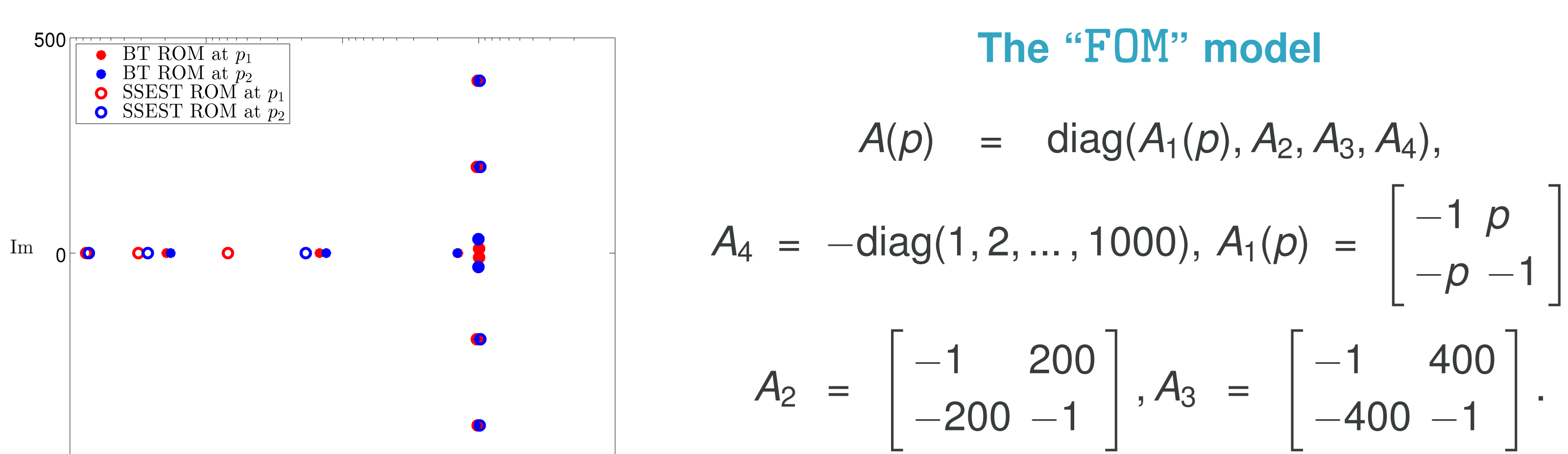

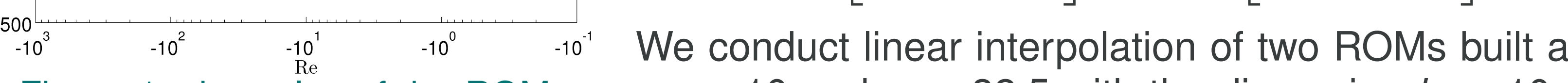
Figure 1: the poles of the ROMs $\quad p=10$ and $p=32.5$ with the dimension $k=10$.

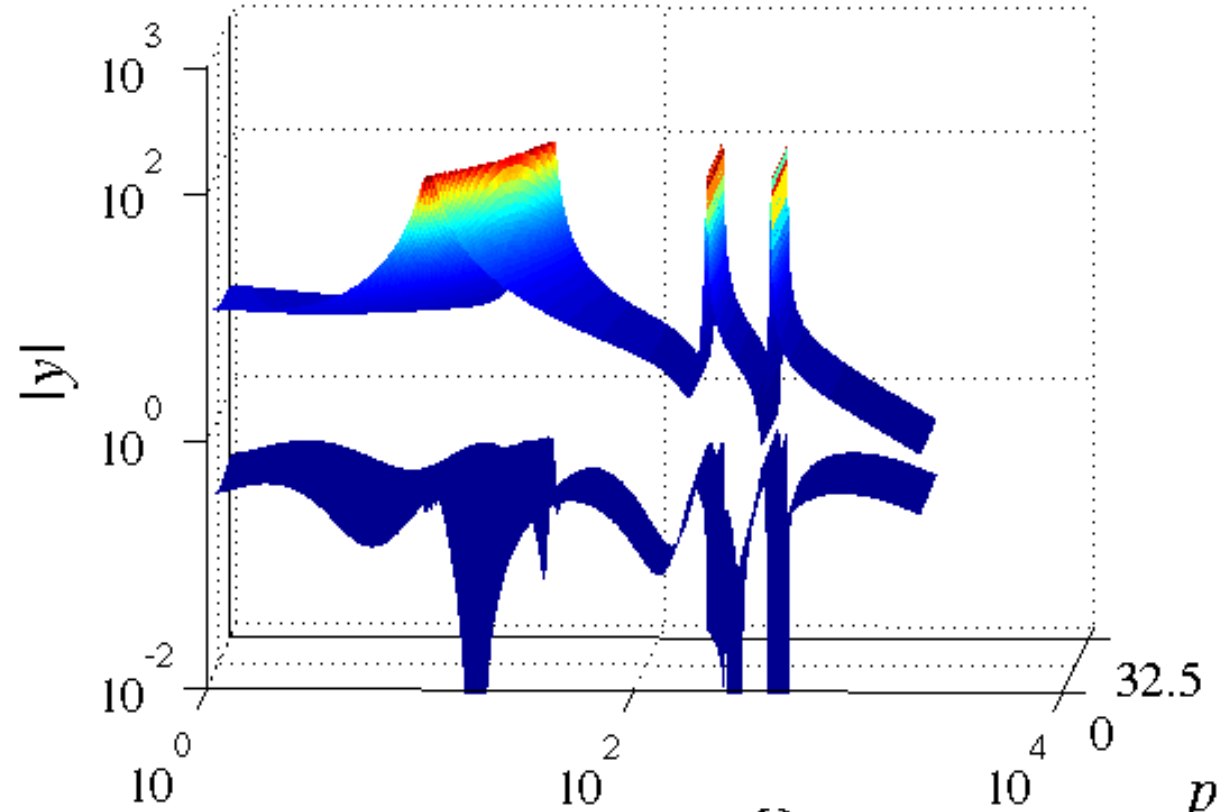

Figure 2: Interpolation of two BT ROMs

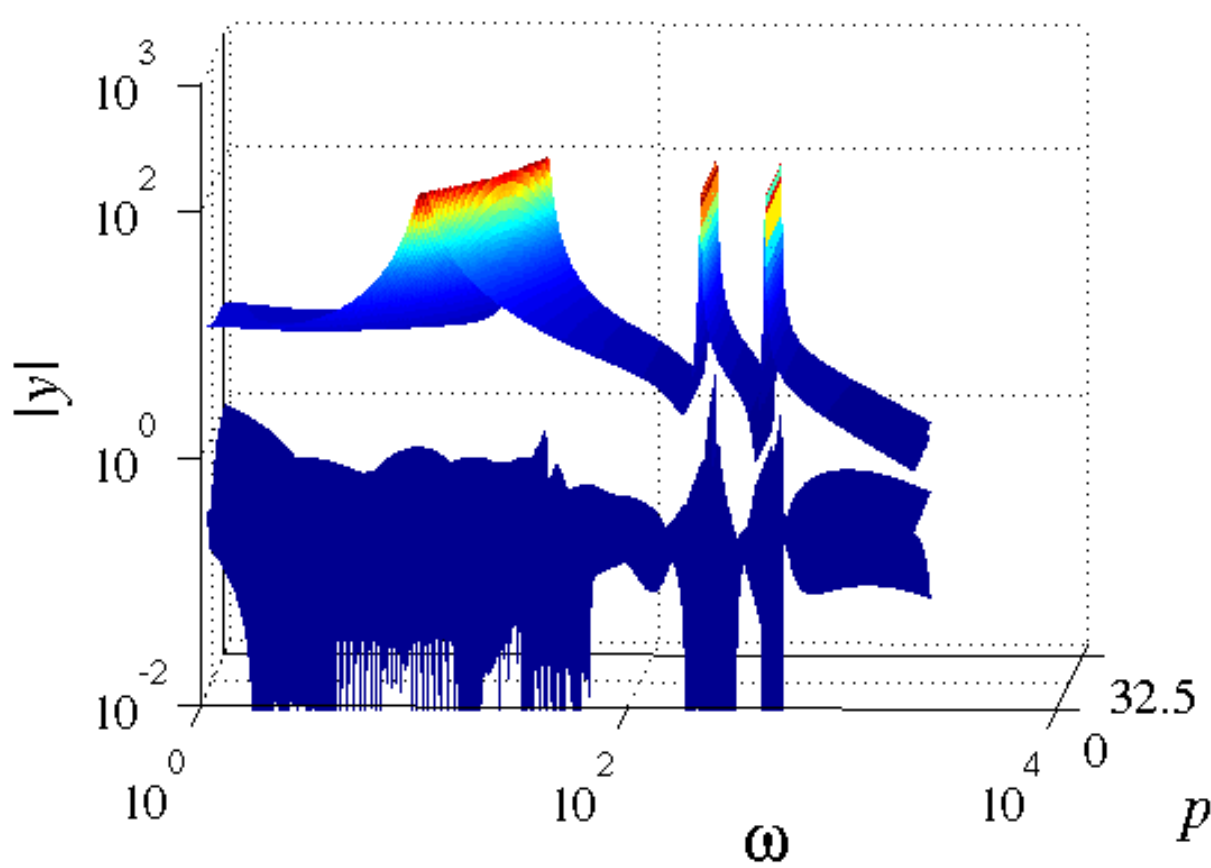

Figure 4: Interpolation of a BT ROM built at $=10$ and a ssest ROM built at $P=32.5$

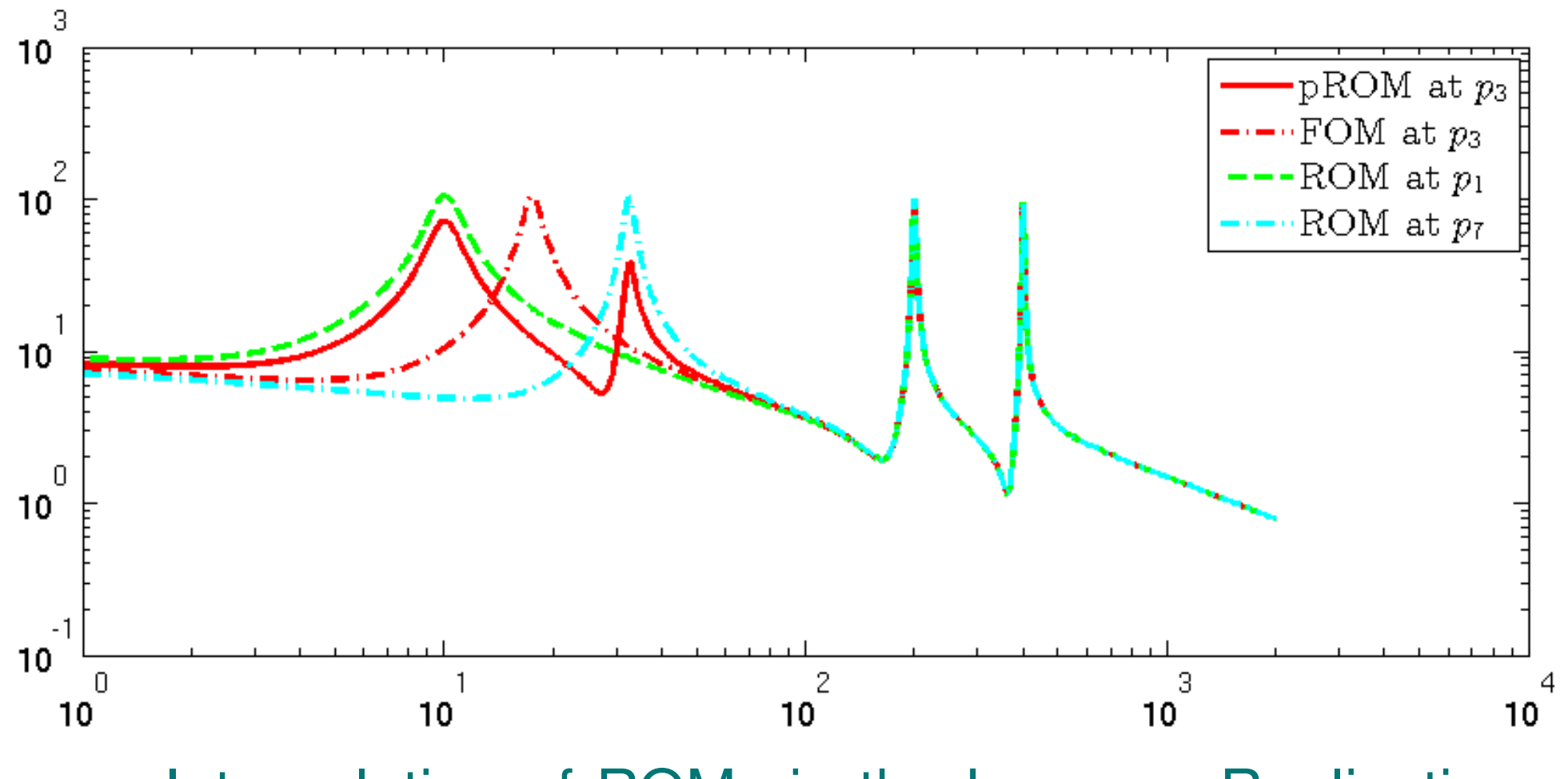

Reference Figure: Interpolation of ROMs in the Loewner Realization. Fixed Poles! Conclusions

(1) The realization problem plays a central role in the interpolation of ROMs

(2) Interpolating ROMs in MMR is equivalent to interpolating the positions and amplitudes of poles. (3) It gives accurate results even when the ROMs are built by different methods: the errors of the interpolated ROMs is comparable to those of the ROMs used for interpolation

(4) It has low computational cost, capable of dealing with complicated parameter dependence, and is relatively insensitive to the dimension of the parameter space. 